\title{
ON THE PRINCIPLE OF COMBINATION AND STOKES' LAW IN THE X-RAY SERIES.
}

\author{
By D. Coster.
}

SYNOPSIS.

New X-Ray Line $\gamma_{6}$ for Elements Tantalum to Uranium.-The two emission lines $\gamma_{2}$ and $\gamma_{3}$ are found to be in reality three lines. The new line $\gamma_{6}$ has wave-lengths of IIIO, I072, 842, 630 and $593 \times 10^{-11} \mathrm{~cm}$. for Ta, W, Tl, Th and U respectively. In the cases of $\mathrm{Ir}, \mathrm{Pt}$ and $\mathrm{Au}$ it is too near $\gamma_{2}$ and in the cases of $\mathrm{Pb}$ and $\mathrm{Bi}$ too near $\gamma_{3}$ to be separated.

Nerw Absorption Lines of the $M$-series.- $\mathrm{Ma}_{1}, \mathrm{Ma}_{2}$ and $\mathrm{Ma}_{3}$ have been measured for bismuth, and $\mathrm{Ma}_{4}$ and $\mathrm{Ma}_{5}$ for thorium and uranium, the wave-lengths being, respectively, $4762,4569,3894,257 \mathrm{I}, 2388,2385$ and $2228 \times 10^{-11} \mathrm{~cm}$.

Identification of the Line for Tantalum and Tungsten.-For all the elements Os to $\mathrm{U}$, the difference in wave-lengths of $\beta_{2}-\beta_{5}$ is equal to about $29 \times 10^{-11} \mathrm{~cm}$. If this difference is the same for $\mathrm{Ta}$ and $\mathrm{W}$ also, the $\beta_{5}$ lines for these elements must be the lines I220.8 and I250.6, respectively, instead of those ordinarily assumed.

Measurement of $L a$, and $L a_{2}$ for Tungsten by a different method gave the same wave-lengths as those found by Duane and Patterson.

Relationships between Emission and Absorption X-Ray Spectra.-(I) Stokes'slaw seemed not to hold in the case of W. It was thought the apparent discrepancies might be due to an error in the measurement of $\mathrm{La}_{1}$ and $\mathrm{La}_{2}$, but these measurements were found correct; however, the discrepancies disappear if we identify $\beta_{5}$ with I220.8 instead of $\mathrm{I}_{2} \mathrm{I}_{2} .5$ and if we associate $\gamma_{2}$ and $\gamma_{3}$ with $\mathrm{La}_{3}$ and the new line $\gamma_{6}$ with $\mathrm{La}_{2}$. (2) Relations between frequencies. The frequencies of seven of the L-series lines are each approximately equal to the difference between the frequency of an La-line and that of an Ma-line.

I $\mathrm{N}$ a previous note I called attention to the fact that Stokes' law holds for the L-series of the elements $\mathrm{Pt}-\mathrm{U}$. For W however the limiting wave-lengths $\mathrm{La}_{1}$ and $\mathrm{La}_{2}$ seemed to be much larger than the wave-lengths $\beta_{5}$ and $\gamma_{2}$ respectively; and I suggested a possible source of error in the method by which Duane and Patterson determined the absorption-wave-lengths of tungsten. On account of the great theoretical importance of this matter I have repeated the measurements, but with essentially the same result as these authors. To avoid every kind of complication I proceeded in the following manner. Use was made of a water-cooled bulb with aluminium-cathode, constructed by Prof. Siegbahn and described by A. Hadding. ${ }^{1}$ This bulb has the great advantage

${ }^{1}$ Zeitschrift für Physik, 3, 369, I920. 
that the slit of the spectrograph can be placed very near to the anticathode. It was driven with a current up to $15 \mathrm{~mA}$ and a tension of about $40 \mathrm{kV}$. First the absorption spectrum was taken with an iron anticathode and the absorbing substance placed between the bulb and the slit. Part of the slit was covered with a thick lead screen, so that only the upper part of the plate was exposed. In order to eliminate effects due to inhomogeneities of the absorbing screen, this was turned round during the exposure. Then the iron anticathode was replaced by a tungsten one, the absorbing substance was removed and by a suitable adjustment of the lead screen an emission-spectrum could be obtained on the lower part of the same plate. The plates demonstrated quite obviously that the absorption wave-lengths are considerably larger than the wave-lengths thus far denoted by $\beta_{5}$ and $\gamma_{2}$. For purposes of control the $\mathrm{La}_{2}$ and the $\mathrm{L} \gamma$-lines of gold and lead were taken in the same way; but, as might be expected, no such a difference was found here.

Now we may escape from the theoretical difficulty lying in ân excepitional condition of the tungsten-spectrum by assuming, as already has been done by Hoyt ${ }^{1}$ and by Dauvillier, ${ }^{2}$ that the $\beta_{5}$-line of this element has not as yet been identified accurately. As may be seen from Table I.

TABle $I^{3}{ }^{3}$

\begin{tabular}{c|c|c|c|c|c|c|c|c|c|c|c}
\hline & U. & Th. & Bi. & Pb. & Tl. & Au. & Pt. & Ir. & Os. & W. & Ta. \\
\hline$\beta_{2}-\beta_{5} \ldots$ & 28.58 & 28.49 & 30.63 & 30.48 & 29.56 & 29.55 & 29.40 & 29.87 & 28.38 & 29.41 & 30.32 \\
\hline
\end{tabular}

the wave-length difference $\beta_{2}-\beta_{5}$ is nearly constant from $U$ down to Os. If now we take for $\beta_{5}$ of $\mathrm{W}$ the line I21 2.8 and for Ta the line 1250.6 then for these elements the difference $\beta_{2}-\beta_{5}$ likewise remains nearly the same.

As regards the line $\gamma_{2}$, the situation is more complicated than could be anticipated formerly. Recently I have taken new photographs of the emission-lines of the elements $\mathrm{Ta}-\mathrm{U}$ with the same metal bulb. ${ }^{4} \mathrm{I}$ find, that instead of the lines thus far denoted by $\gamma_{2}$ and $\gamma_{3}$, we must assume three lines. ${ }^{5}$ One of these, which we may call $\gamma_{6}$, lies at a constant wave-length-distance from $\gamma_{1}$. The frequency-difference $\gamma_{\gamma^{6}}-\gamma_{\beta 5}$ is

1 Proc. Nat. Ac. Sc. Washington, Nov., I920.

2 Comptes Rendus, iI April, r921.

3 The wave-lengths are given in $\mathrm{cm} . \times 10^{-11}$.

${ }^{4}$ See Zeitschrift für Physik, 6, I 58, I92 I.

${ }^{5}$ An analogous hypothesis was pronounced some time ago in a letter from Mr. Wentzel to the author. 
the same as the difference $\gamma_{\beta 1}-\gamma_{a 2}$. For the elements $\mathrm{Ta}$ and $\mathrm{W}$, $\gamma_{6}$ is of very small intensity relatively to the other $\gamma$-lines; but, in the neighborhood of $\mathrm{Pt}$ there is a sudden rise in its intensity. A similar change has been observed for $\beta_{5}$. Moreover, there are two lines $\gamma_{2}$ and $\gamma_{3}$, which lie at about the same wave-length-distance from one another and which both have about the same intensity. Only for the elements Ta, W, Tl and $U$ was $\gamma_{6}$ separated from the lines $\gamma_{2}$ and $\gamma_{3}$, but where complete separation did not occur, a corresponding broadening and darkening of $\gamma_{2}$ or of $\gamma_{3}$ could usually be observed.

TABLE II.

\begin{tabular}{|c|c|c|c|c|c|c|}
\hline & $\gamma_{1}$ & $\gamma_{6}$ & $\gamma_{2}$ & $\gamma_{3}$ & $\gamma_{1}-\gamma_{6}$ & $\gamma_{2}-\gamma_{3}$ \\
\hline $\mathrm{U}$ & 612.83 & 592.6 & 604.4 & 597.0 & - 20.23 & 7.4 \\
\hline Th. . & 651.03 & 630.1 & & & 20.93 & \\
\hline Bi $\ldots \ldots \ldots$ & 810.65 & 787.4 & 792.9 & 787.4 & 23.25 & 5.5 \\
\hline $\mathrm{Pb} \ldots .$. & 837.08 & 813.70 & 818.2 & 813.70 & 23.38 & 4.5 \\
\hline $\mathrm{T} 1 \ldots$ & 865.29 & 841.7 & 844.7 & 837.9 & 23.59 & 6.8 \\
\hline $\mathrm{Au} \ldots$ & 924.37 & 901.25 & 901.25 & 859.68 & 23.12 & 5.57 \\
\hline Pt... & 955.45 & 931.7 & 931.7 & 925.6 & 23.75 & 6.1 \\
\hline Ir. & 988.41 & 963.6 & 963.6 & 956.6 & 24.8 & 7.0 \\
\hline W. . & $1,095.53$ & $1,072.0$ & $1,065.84$ & $1,059.65$ & 23.53 & 6.19 \\
\hline Ta... & $1,134.71$ & $1,110.0$ & $1,102.0$ & $1,096.2$ & 24.71 & 5.8 \\
\hline
\end{tabular}

In Table II. the wave-lengths of the lines $\gamma_{1}, \gamma_{6}, \gamma_{2}$ and $\gamma_{3}$ and the wave-length-differences $\gamma_{1}-\gamma_{6}$ and $\gamma_{2}-\gamma_{3}$ are given in $\mathrm{cm}$. IO ${ }^{-11}$. It seems to be most probable that only $\gamma_{6}$ is connected with the $\mathrm{La}_{2}$ discontinuity, whereas $\gamma_{2}$ and $\gamma_{3}$ both belong to $\mathrm{La}_{3}$.

Thus far only three absorption-discontinuities $\mathrm{Ma}_{1}, \mathrm{Ma}_{2}$ and $\mathrm{Ma}_{3}$ in the M-series of $\mathrm{U}$ and $\mathrm{Th}$ were known, these corresponding to the measurements of Stenström. I have succeeded in measuring two other discontinuities $\mathrm{Ma}_{4}$ and $\mathrm{Ma}_{5}$ of shorter wave-length of $\mathrm{U}$ and $\mathrm{Th}$ and $\mathrm{Ma}_{1}, \mathrm{Ma}_{2}$ and $\mathrm{Ma}_{3}$ of $\mathrm{Bi}$, with the following provisional results:

TABLE III.

\begin{tabular}{l|c|c|c|c|c}
\hline & $\mathbf{M a}_{1}$ & $\mathbf{M a}$ & $\mathbf{M a}_{3}$ & $\mathbf{M a}_{4}$ & $\mathbf{M a}_{\mathbf{5}}$ \\
\hline $\mathrm{U} \ldots \ldots \ldots \ldots \ldots \ldots$ & 3,491 & 3,326 & 2,873 & 2,385 & 2,228 \\
$\mathrm{Th} \ldots \ldots \ldots \ldots \ldots$ & 3,721 & 3,552 & 3,058 & 2,571 & 2,388 \\
$\mathrm{Bi} \ldots \ldots \ldots \ldots \ldots$ & 4,762 & 4,569 & 3,894 & & \\
\hline
\end{tabular}

The values for $\mathrm{Ma}_{1}, \mathrm{Ma}_{2}$ and $\mathrm{Ma}_{3}$ of $\mathrm{U}$ and $\mathrm{Th}$ were taken from Stenström's dissertation. As is seen from Table IV. the frequencies of the lines $1, \eta, \alpha_{1}, \alpha_{2}, \beta_{1}, \beta_{3}$ and $\beta_{4}$ are each equal to the difference of an Labsorption-frequency and a M-absorption-frequency. 
TABLE IV. ${ }^{1}$

\begin{tabular}{|c|c|c|c|c|c|c|c|c|c|c|}
\hline & 1 & $\mathrm{~L} \alpha_{1}-\mathrm{M} \alpha_{5}$ & $\eta$ & $\mathbf{L} \alpha_{2}-\mathbf{M} \alpha_{5}$ & $\alpha_{1}$ & \multicolumn{2}{|c|}{$\mathbf{L} \alpha_{1}-\mathbf{M} \alpha_{1}$} & \multicolumn{2}{|c|}{$\alpha_{2}$} & $L \alpha_{1}-\mathbf{M} \alpha_{2}$ \\
\hline $\begin{array}{l}\text { U....... } \\
\text { Th..... } \\
\text { Bi...... }\end{array}$ & $\begin{array}{l}855.84 \\
819.19\end{array}$ & $\begin{array}{l}855.1 \\
818.8\end{array}$ & $1,134.95$ & $1,131.8$ & $\begin{array}{r}1,003.23 \\
\cdot 955.78 \\
789.54\end{array}$ & \multicolumn{2}{|c|}{$\begin{array}{r}1,002.17 \\
954.80 \\
797.43\end{array}$} & \multicolumn{2}{|c|}{$\begin{array}{l}990.37 \\
944.08 \\
790.20\end{array}$} & $\begin{array}{l}989.21 \\
943.15 \\
789.35\end{array}$ \\
\hline & & $\beta_{1}$ & $\mathrm{~L} \alpha_{2}-\mathrm{M} \alpha_{2}$ & $\beta_{3}$ & \multicolumn{2}{|c|}{$\mathbf{L} \alpha_{3}-\mathbf{M} \alpha_{3}$} & \multicolumn{2}{|c|}{$\beta_{4}$} & \multicolumn{2}{|c|}{$\mathrm{L}_{\alpha_{3}-\mathrm{M}} \alpha_{4}$} \\
\hline \multicolumn{2}{|l|}{ U. . } & $1,269.08$ & $1,265.87$ & $1,286.29$ & \multicolumn{2}{|c|}{$1,285.78$} & \multicolumn{2}{|c|}{$1,222.53$} & \multicolumn{2}{|c|}{$1,220.86$} \\
\hline \multirow{2}{*}{\multicolumn{2}{|c|}{$\begin{array}{l}\text { Th. } \\
\text { Bi. }\end{array}$}} & $1,194.94$ & $1,193.15$ & $1,211.67$ & \multicolumn{2}{|c|}{$1,209.77$} & \multicolumn{2}{|c|}{$1,155.00$} & \multirow{2}{*}{\multicolumn{2}{|c|}{$1,151.86$}} \\
\hline & & 959.93 & 958.16 & 973.85 & 975. & & & & & \\
\hline
\end{tabular}

Apart from the accidental errors there seems to be a systematic deviation between the line-frequency and the difference of the absorptionfrequencies, so that we may conclude that there must be a small systematical error either in my measurements of the lines or in the work of Duane and Patterson. If this is the case, it is most probable that the same error should be found in the measurements of all the elements. We should therefore be inclined to conclude that for the elements $\mathrm{Pt}$, $\mathrm{Au}, \mathrm{Tl}, \mathrm{Pb}$ and $\mathrm{Bi}$, and perhaps for $\mathrm{W}$ also, the absorption-wave-lengths are a little shorter than the corresponding wave-lengths $\beta_{5}$ and $\gamma_{6}$ and that the difference should correspond to a difference of about 5-Io volts.

A superficial examination of my plates does not give the impression that the absorption-wave-lengths are shorter than the corresponding emission-wave-lengths. But the selective absorption commences with a faint white line, which may be tentatively considered as an image of the slit just as the black emission-lines are. This shows that the electron "prefers" the absorption of a very definite wave-length, which is at the same time the smallest frequency it is able to absorb. To estimate this frequency we are to measure the middle of the white absorption-line relatively to the middle of the black emission-lines. I hope to have later the opportunity to study this problem with the required accuracy with the photometer-method.

It gives me great pleasure to express my thanks to Professor Siegbahn, who put the apparatus of his laboratory at my disposal and advanced the work by his great interest.

Lund, Sweden, Phys. Lab. Of the University, June, I92I.

1 The frequencies have been calculated in multiples of the Rydberg constant (limiting frequency of the Lyman series of hydrogen). 